\title{
QUALIDADE DE VIDA DOS PROFISSIONAIS DA ATENÇÃO PRIMÁRIA EM SAÚDE
}

\section{QUALITY OF LIFE OF PRIMARY HEALTH CARE PROFESSIONALS}

\author{
Koch, J. S. R ${ }^{1}$, Menetrier, J. V. ${ }^{1}$, Zonta, F. N. S. ${ }^{1}$ \\ Afiliações: 1- Universidade Paranaense, campus de Francisco Beltrão. \\ Endereço do autor de correspondência Av. Júlio Assis Cavalheiro, 2000, Centro, Francisco Beltrão, Paraná, Brasil. CEP: 85601-000. \\ Franciele.ns@prof.unipar.br
}

\section{Resumo}

Objetivou-se avaliar a qualidade de vida dos profissionais atuantes nas Estratégias de Saúde da Família, sendo este um estudo de campo, quantitativo, com aplicação do questionário WHOQOL-bref. Os dados foram coletados de junho a agosto de 2019 e analisados pelo SPSS 25.0. Obteve-se uma participação de 200 funcionários, sendo 190 do sexo feminino. Em relação à qualidade de vida no geral, a mesma foi classificada como regular, por 148 profissionais. Na avaliação dos domínios separadamente, os mesmos também obtiveram o resultado regular. Entre os quatro domínios avaliados, o domínio físico obteve a menor média, de 57,58 . Em contrapartida, a maior média foi do domínio social, 68,75. Percebeu-se, ainda, que um elevado número (63) de pesquisados relatou fazer uso de alguma medicação psicotrópica, sendo 51 da classe antidepressiva. Denota-se a necessidade de intervenções para este público, pois um serviço de qualidade só é ofertado quando há uma boa qualidade de vida associada a boas condições de saúde física e psicológica.

Palavras-chave: saúde do trabalhador; pessoal de saúde; atenção básica de saúde.

\section{Abstract}

The quality of life evaluation of acting professionals in Family Health Strategies was the objective of this quantitative field study, with the application of the WHOQOL-bref questionnaire. Data collection took place from June to August 2019 and SPSS 25.0 performed data analysis. Of the 200 employees who participated in the study, 190 were female. A number of 148 professionals rated the quality of life as regular. In the separate evaluation of the domains, they also obtained a regular result. Among the four evaluated domains, the physical domain obtained the lowest average, 57.58 . By contrast, the highest average was the social domain, corresponding to 68.75. It was also noticed that a high number (63) of interviewed reported using some psychotropic medication, being 51 of the antidepressant class. This public need interventions, because the offer of a quality service only happens when there is a good quality of life associated with good physical and psychological health.

Keywords: worker's health; health personnel; primary health care. 


\section{Introdução}

O termo Qualidade de Vida (QV) é de difícil conceituação, já que cada indivíduo á avalia em contextos diferentes. A Organização Mundial da Saúde (OMS) define QV como o conhecimento que a pessoa tem sobre si próprio, inerente ao meio em que vive, e ainda sugere seis domínios relacionados à QV: Saúde Física, Saúde Psicológica, Nível de Independência, Relações Sociais, Meio Ambiente e Valores e Crenças Pessoais ${ }^{1}$.

A QV se caracteriza pelo bem-estar do indivíduo em todos os aspectos abrangentes da sua vida. Ela se dá, inclusive, pela realização em seu espaço laboral, pois é onde o indivíduo, na maioria das vezes, passa a maior parte de seu dia. Consequentemente, a satisfação dele em relação às suas prioridades diárias, influenciará a sua qualidade de vida ${ }^{2}$

O trabalho se caracteriza como uma transformação que o homem executa conforme as suas necessidades, se tornando uma autorrealização pessoal ${ }^{3}$. Trata-se de um objeto essencial para o crescimento e desenvolvimento humano, uma condição necessária, natural, que o afirma na sociedade ${ }^{4}$.

Entretanto, é sabido que o trabalho em saúde, tende a ser mais exaustivo, e com isso, os profissionais estão mais propensos à insatisfação, medos, adoecimentos e ao sentimento de incapacidade ${ }^{5}$. Além disso, por vezes, carregam a responsabilidade de encontrar formas de estimular a população a buscar melhores condições de vida, elaborando planos e estratégias para atender a demanda existente ${ }^{6}$.

As alterações na QV dos profissionais em saúde implicam na sua rotina, na própria assistência ao usuário e na equipe multiprofissional, o que pode ser considerado um problema de saúde pública ${ }^{6}$.

Diante do pressuposto, percebe-se a necessidade de estudos que envolvam tal público, visto que estes profissionais sofrem de uma grande demanda psicológica e física no próprio ambiente de trabalho, que pode interferir em seus afazeres diários.

Neste ambiente, a má QV gera absenteísmo, maior número de acidentes no trabalho, tensão muscular, apatia, cefaleia, taquicardia, desinteresse no desenvolvimento profissional, estresse, depressão e alterações no sono $^{7}$. Além disso, estes profissionais podem apresentar menor produtividade e qualidade no serviço prestado $^{8}$, por conta dos agravamentos da saúde física e mental, quanto, pelo recorrente aumento no uso de psicofármacos ${ }^{9}$ 
Assim, a adequada assistência prestada pelos profissionais de saúde à sociedade, depende diretamente de uma base de profissionais satisfeitos e com QV apropriada, conforme constata estudo realizado no Rio Grande do $\mathrm{Sul}^{6}$. Desse modo, a realização da presente pesquisa possibilita identificar a $\mathrm{QV}$ dos profissionais atuantes nas Estratégias de Saúde da Família (ESF) em município do interior do Paraná, e gera dados para ações que possam melhorar a vida pessoal e atuação profissional dos pesquisados, resultando em atendimentos ao público, ainda mais humanizados ${ }^{6}$.

Diante do exposto, o objetivo desta pesquisa foi avaliar a qualidade de vida dos profissionais atuantes nas ESF, bem como os fatores sociais relacionados.

\section{Métodos}

Trata-se de um estudo de campo, exploratório, descritivo, com abordagem quantitativa. A pesquisa ocorreu junto a equipes da ESF da região urbana de um município de médio porte do Paraná, constituídas por 273 funcionários atuando no setor, na ocasião da pesquisa.

$$
\text { A amostra por conveniência foi }
$$

constituída por 200 (duzentos) funcionários das ESF, incluindo: médicos, enfermeiros, farmacêuticos, odontólogos, técnicos e auxiliares de enfermagem, Agente Comunitário de Saúde (ACS), Agente de Combate de Endemias (ACE), assistentes administrativos, serviços gerais e auxiliares e/ou técnicos em saúde mental.

A coleta de dados ocorreu entre 10 de junho de 2019 e 08 de agosto de 2019, por meio da aplicação de questionários. Primeiramente foi requisitada à Secretaria Municipal de Saúde a autorização para a pesquisa e coleta de dados. Posteriormente, foi realizado o agendamento da visita em cada unidade com o enfermeiro. No dia da coleta, todos os funcionários foram para a sala de reuniões, onde foram expostos o objetivo da pesquisa, os benefícios e o anonimato do pesquisado, além da descrição dos questionários. Os participantes receberam o Termo de Consentimento Livre e Esclarecido (TCLE). Aqueles que aceitaram participar da pesquisa, o assinaram, e em seguida, foram convidados a responder dois instrumentos de coleta de dados (questionários).

$\mathrm{O}$ primeiro questionário de caráter sociodemográfico, semiestruturado, avaliou o 
sexo, idade, estado marital, tempo de serviço, renda familiar mensal, escolaridade, função exercida, realização de atividade física, religião, outros vínculos empregatícios, quantas horas semanais o mesmo trabalha, se exerce a sua profissão no período noturno, doenças de base e uso de medicações psicoativas. Este é um questionário próprio elaborado pelas pesquisadoras, baseado em literatura pertinente.

O segundo instrumento é validado pela OMS para caracterizar/classificar a qualidade de vida do pesquisado. O WHOQOL-bref é constituído por 26 questionamentos sobre a percepção do indivíduo acerca da sua qualidade de vida e ainda faz uma separação da qualidade de vida em domínios, sendo esses: físico, psicológico, relações sociais e meio ambiente, avaliando-os separadamente. Trata-se de uma adaptação do questionário WHOQOL-100 ${ }^{10}$. Os domínios são avaliados considerando pontuações até 2,9 como ruim, de 3,0 a 3,9 regular, entre 4,0 e 4,9 boa, e 5, muito boa. As médias dos domínios foram convertidas numa escala de 0 a 100, conversão essa que é indicada pelo instrumento, considerando que, quanto maior o valor, melhor a qualidade de vida ${ }^{11}$.

A análise dos dados com frequência descritiva, foi realizada por meio do Software
Statistical Package of Social Sciences for Windows (SPSS) 25.0.

Esta pesquisa segue todos os preceitos éticos e legais, regulamentados pela resolução 466/2012 do Conselho Nacional de Saúde/ Ministério da Saúde. Para sua realização obtevese a declaração de permissão para utilização de dados e a aprovação do Comitê de Ética em Pesquisa da Universidade Paranaense - UNIPAR sob o protocolo $\mathrm{n}^{\mathrm{o}} 3.364 .056 / 2019$.

\section{Resultados}

Entre os 200 participantes, 95,0\% eram do sexo feminino, e a maioria trabalhava há menos de 5 anos no serviço $(45,0 \%)$. Houve prevalência de casados $(61,5 \%)$ e com dois filhos $(37,0 \%)$. A renda familiar prevalente foi de 3 a 4 salários mínimos (41,0\%). Em relação à escolaridade, 43,0\% possuíam o ensino superior completo e $40,0 \%$ ensino médio completo. A religião católica foi referida por $74,0 \%$ da amostra. Quanto à profissão exercida pelos pesquisados, a maioria $(46,0 \%)$ eram ACS e ACE, seguida por técnicos e auxiliares de enfermagem (14,0\%). Quanto ao trabalho noturno e mais de um vínculo empregatício, apenas $12,0 \%$ e $11,0 \%$ respectivamente, se 
enquadravam nesta condição, conforme descrito na (tabela 1).

Tabela 1. Dados referentes ao Perfil sociodemográfico dos profissionais da equipe da Estratégia de Saúde da Família de um Município do Paraná em 2019.

\begin{tabular}{|c|c|c|}
\hline VARIÁVEIS & n (200) & $(\%)$ \\
\hline \multicolumn{3}{|l|}{ Sexo } \\
\hline Feminino & 190 & 95,0 \\
\hline Masculino & 10 & 5,0 \\
\hline \multicolumn{3}{|l|}{ Tempo de serviço } \\
\hline$<5$ anos & 90 & 45,0 \\
\hline 5 a 10 anos & 72 & 36,0 \\
\hline$>10$ anos & 38 & 19,0 \\
\hline \multicolumn{3}{|l|}{ Situação conjugal } \\
\hline Casado & 123 & 61,5 \\
\hline Solteiro & 47 & 23,5 \\
\hline Viúvo & 6 & 3,0 \\
\hline Divorciado & 24 & 12,0 \\
\hline \multicolumn{3}{|l|}{$\mathbf{N}^{\circ}$ de filhos } \\
\hline Sem Filhos & 47 & 23,5 \\
\hline Um Filho & 56 & 28,0 \\
\hline Dois Filhos & 74 & 37,0 \\
\hline Três Filhos & 19 & 9,5 \\
\hline Quatro ou mais filhos & 4 & 2,0 \\
\hline \multicolumn{3}{|l|}{ Renda familiar } \\
\hline Até 1 Salário Mínimo & 11 & 5,5 \\
\hline 1 a 2 Salários Mínimos & 53 & 26,5 \\
\hline 3 a 4 Salários Mínimos & 82 & 41,0 \\
\hline > 4 Salários Mínimos & 54 & 27,0 \\
\hline \multicolumn{3}{|l|}{ Religião } \\
\hline Católico & 148 & 74,0 \\
\hline Evangélico & 37 & 18,5 \\
\hline Outra & 7 & 3,5 \\
\hline Nenhuma & 8 & 4,0 \\
\hline \multicolumn{3}{|l|}{ Escolaridade } \\
\hline Ensino Médio Incompleto & 4 & 2,0 \\
\hline Ensino Médio Completo & 80 & 40,0 \\
\hline Ensino Superior Incompleto & 30 & 15,0 \\
\hline
\end{tabular}

$\begin{array}{lcc}\text { Ensino Superior Completo } & 86 & 43,0 \\ \text { Função } & & \\ \text { Médico } & 13 & 6,5 \\ \text { Enfermeiro } & 14 & 7,0 \\ \text { Técnico/Auxiliar Enfermagem } & 28 & 14,0 \\ \text { ACS e ACE } & 92 & 46,0 \\ \text { Administrativo } & 18 & 9,0 \\ \text { Serviços Gerais } & 9 & 4,5 \\ \text { Dentista } & 9 & 4,5 \\ \text { Auxiliar de Dentista } & 15 & 7,5 \\ \text { Farmacêutico e atendente de } & 2 & 1,0\end{array}$

farmácia

\section{Outros vínculos empregatícios}

\begin{tabular}{lcc} 
Sim & 24 & 12,0 \\
Não & 176 & 88,0 \\
\hline Fonte: Dados da pesquisa, 2019. &
\end{tabular}

Referente aos hábitos de vida, 55,5\% dos participantes relataram não praticar atividades físicas e 18,0\% informaram possuir alguma doença de base, sendo a Hipertensão Arterial Sistêmica (HAS) a mais citada em 38,8\%. Em relação ao uso de medicação para saúde mental, 31,5\% referiram tal prática. A classe medicamentosa mais observada foi a de antidepressivos $(80,9 \%)$, sendo a fluoxetina $(26,9 \%)$ e a sertralina $(25,3 \%)$ as mais descritas. Dos profissionais que fazem o uso de medicações para saúde mental, tem-se o destaque das mulheres, com 96,8\% de uso. Quanto a classe profissional, os ACS e ACE encontram-se em primeiro lugar com 47,6\%, seguido pelas classes de técnico de enfermagem e assistente administrativo com 12,6\%, após os 
enfermeiros e serviços gerais, tendo um uso de $7,9 \%$ (tabela 2).

Tabela 2. Hábitos de vida, doenças associadas e uso de medicações pelos profissionais da equipe Estratégia de Saúde da Família de um Município do Paraná em 2019.

\begin{tabular}{|c|c|c|}
\hline VARIÁVEL & n (200) & $(\%)$ \\
\hline \multicolumn{3}{|c|}{ Realização de atividade física } \\
\hline Sim & 89 & 44,5 \\
\hline Não & 111 & 55,5 \\
\hline \multicolumn{3}{|c|}{ Trabalho em período noturno } \\
\hline Sim & 22 & 11,0 \\
\hline Não & 178 & 89,0 \\
\hline \multicolumn{3}{|l|}{ Doenças de base } \\
\hline Sim & 36 & 18,0 \\
\hline Não & 164 & 82,0 \\
\hline \multicolumn{3}{|l|}{ Doenças de base citadas } \\
\hline HAS & 14 & 38,8 \\
\hline Outros & 22 & 61,1 \\
\hline \multicolumn{3}{|c|}{ Uso de medicação para saúde mental } \\
\hline Sim & 63 & 31,5 \\
\hline Não & 137 & 68,5 \\
\hline \multicolumn{3}{|l|}{ Medicamentos listados } \\
\hline Fluoxetina & 17 & 26,9 \\
\hline Sertralina & 16 & 25,3 \\
\hline Outros & 30 & 47,6 \\
\hline \multicolumn{3}{|c|}{ Classe medicamentosa consumida } \\
\hline Antidepressivo & 51 & 80,9 \\
\hline Outros & 12 & 19,0 \\
\hline \multicolumn{3}{|c|}{ Uso de medicamento listados por gênero } \\
\hline Feminino & 61 & 96,8 \\
\hline Masculino & 2 & 3,1 \\
\hline \multicolumn{3}{|c|}{ Uso de medicamentos listados por classe profissional } \\
\hline ACS E ACE & 30 & 47,6 \\
\hline Técnicos de Enfermagem & 8 & 12,6 \\
\hline Assistente Administrativo & 8 & 12,6 \\
\hline Enfermeiros & 5 & 7,9 \\
\hline Serviços Gerais & 5 & 7,9 \\
\hline Demais Profissionais & 7 & 11,1 \\
\hline
\end{tabular}

Fonte: Dados da pesquisa, 2019.
Ao analisar a qualidade de vida destes profissionais verificou-se que apresentaram uma média regular referente à qualidade de vida. Considerando a qualidade de vida em uma totalidade, obteve-se uma média de 68,81 , e vale ressaltar que o valor mínimo foi de 12,50, e 100 para máximo (tabela 3).

Tabela 3. Médias de cada domínio da qualidade de vida dos profissionais da equipe da Estratégia de Saúde da Família de um Município do Paraná em 2019, a partir dos escores do WHOQOL-bref $(n=200)$.

\begin{tabular}{lccc}
\hline Domínio & Média & Mínimo & Máximo \\
\hline Físico & 57,58 & 35,71 & 82,14 \\
Psicológico & 62,64 & 20,83 & 95,83 \\
Relações Sociais & 68,75 & 16,67 & 100,00 \\
Meio Ambiente & 62,22 & 21,88 & 87,50 \\
QV no Geral & 68,81 & 12,50 & 100,00 \\
\hline Fonte: Dados da pesquisa, 2019. & &
\end{tabular}

O domínio físico atingiu a menor média de todas as facetas com 57,58, seguido pelo domínio meio ambiente com 62,22. O psicológico obteve uma média de 62,64. A maior média dos quatro domínios foi a de relações sociais, com uma média de 68,75. Entre as quatro facetas apresentadas a menor pontuação foi a de relações sociais com 16,67 , que obteve a maior pontuação em 100, sendo que nenhum dos outros atingiu essa pontualidade. 
Com relação aos domínios pesquisados, as respostas dos profissionais foram divididas em: ruim, regular e boa/muito boa, sendo em todos os domínios, citada com mais frequência classificação regular (tabela 4).

Tabela 4. Número de pesquisados para cada classificação do domínio avaliado pelo WHOQOL-bref $(\mathrm{n}=200)$.

\begin{tabular}{lccc}
\hline Domínio & $\begin{array}{c}\text { Ruim } \\
(\mathbf{n})\end{array}$ & $\begin{array}{c}\text { Regular } \\
(\mathbf{n})\end{array}$ & $\begin{array}{c}\text { Bom/Muito } \\
\text { Bom }\end{array}$ \\
& & & $\mathbf{n}$ \\
\hline Físico & 5 & 194 & 1 \\
Psicológico & 7 & 189 & 4 \\
Relações sociais & 8 & 143 & 49 \\
Meio ambiente & 6 & 184 & 10 \\
QV no Geral & 22 & 148 & 30 \\
\hline
\end{tabular}

Fonte: Dados da pesquisa, 2019.

Na avaliação da QV, no geral, 148 $(74,0 \%)$ pesquisados apontaram a mesma como regular, e 22 pesquisados apontaram a sua qualidade de vida como ruim. O domínio físico foi que obteve o menor número de pessoas que respondeu como bom/muito bom, totalizando 1 pesquisado. Em contrapartida, o domínio social foi o que apresentou maior número de pesquisados respondendo como bom/muito bom, com $49(24,5)$ participantes.

\section{Discussão}

A presente pesquisa aponta como seu principal resultado que a $\mathrm{QV}$ da população pesquisada se encontrava como regular em todos os aspectos abordados pelos instrumentos utilizados para avaliação. Nota-se também um uso desenfreado de medicações psicotrópicas nos profissionais pesquisados, principalmente da classe antidepressiva.

Conforme resultados apresentados vemos que o gênero feminino foi predominante entre os colaboradores da área da saúde, corroborando o estudo de Carlotto com 83,0\%, e o de Moreira com $95,1 \%{ }^{12,13}$. Isto se deve ao fato de que desde os primórdios o cuidado em saúde tem sido realizado por mulheres, concepção essa que apesar de estar sendo modificada, ainda sobressai. Em sua maior parte, os profissionais eram casados ou com companheiros, corroborando um estudo realizado no Rio Grande do Sul, que buscou avaliar as condições de saúde mental dos trabalhadores da ESF, demonstro que $80,2 \%$ tinham uma união estável, prevalecendo também o número de 2 filhos para cada participante em $68,7 \%$. O mesmo autor aponta que a maioria dos pesquisados foram ACS em 57,8\%, seguidos por técnicos de enfermagem em 24,1\%, dados que se assemelham a esta pesquisa ${ }^{13}$. 
O presente estudo apresentou dados semelhantes à pesquisa de Leonelli, na qual a classe trabalhadora de ACS esteve como a mais citada em 86,3\%, seguida por enfermeiro $(75,0 \%)$ e auxiliares de enfermagem $(69,5 \%)^{14}$. Considerando que o ACS realiza visitas domiciliares para todas as famílias, é compreensível o maior número do mesmo em uma unidade de saúde. As atividades desenvolvidas por estes profissionais são regulamentadas pela Emenda Constitucional de $\mathrm{n}^{\circ} 51$ de 14 de fevereiro de 2006 dos $\S \S 4^{\circ}$ e $5^{\circ}$ do art. 198 da Constituição ${ }^{15}$. Os profissionais de enfermagem, colocaram-se o segundo lugar em número de ocupações neste estudo, fato relacionado a grande parte das atividades/demandas existentes em Unidades de Saúde estarem relacionadas com esses profissionais $^{14}$.

A escolaridade preponderante observada foi o ensino superior completo, dado que diverge do estudo de Moreira, no qual o ensino médio completo foi o mais citado em $43,9 \%$ dos pesquisados. Todos os profissionais em saúde devem ter um nível de escolaridade necessário para assumir o cargo, e no estudo em questão, temos o conhecimento de que os pesquisados cumprem o exigido. Isto nos mostra que esta população está em busca de novos saberes, participando de capacitações para o melhor atendimento da comunidade ${ }^{13}$. Este dado corrobora o estudo de Nunes, em que $85,0 \%$ dos pesquisados possuíam uma escolaridade maior que a exigida pelo cargo ocupado ${ }^{16}$.

Em um estudo realizado por Freire, em 2016, muitos profissionais de enfermagem acabaram se afastando do meio laboral, afastamento esse causado por estresse, longas jornadas de trabalho e descanso insuficiente. Isto implica a longo e curto prazo em uma má qualidade de vida ${ }^{17}$. Outro estudo realizado no estado do Rio Grande do Sul apontou que a equipe que constituía o NASF (Núcleo de Apoio à Saúde da Família), apresentou a menor média de qualidade de vida. Este mesmo estudo retratou que os ACS constituíam a classe com a maior qualidade de vida, considerando as exigências de escolaridade para atuar nesta área serem mínimas, do que se pode deduzir que, quanto maior a escolaridade, menor a qualidade de vida ${ }^{6}$.

A religião com maior prevalência foi o catolicismo com $74,0 \%$, fato relacionado ao Brasil ser um país com a maioria da população adepta desta religião. Segundo dados do Instituto Brasileiro de Geografia e Estatística (IBGE), em 
2010, 65,0\% de toda população brasileira eram praticantes do catolicismo ${ }^{18}$.

Quanto ao tempo de serviço, a maioria dos participantes estava atuando nesta área há menos de cinco anos, dados que corroboram com o estudo de Moreira, em que $61,4 \%$ estavam no serviço há menos de cinco anos ${ }^{16}$, mas diverge do estudo realizado com agentes comunitários de saúde, onde a maioria dos profissionais atuavam há mais de dez e menos de quinze anos $(55,19 \%)$, o que se deve à estabilidade dos concursos públicos paras as funções nas quais foram aprovados ${ }^{19}$.

Quando um profissional assume um determinado serviço, o mesmo passa por algumas dificuldades, como a conquista e aproximação do seu público. Um estudo realizado em Palmas, no Tocantins, demonstra que os agentes comunitários em saúde que permanecem um tempo maior em determinado lugar adquirem um entendimento maior da sua finalidade com a sociedade, criando alternativas práticas para a resolução de problemas. Dessa forma, a prática da vivência profissional aumenta a qualidade de vida do indivíduo ${ }^{19}$.

Constatou-se baixa adesão à prática de atividade física dentre os pesquisados $(55,5 \%)$. Este dado se assemelha a um estudo realizado na
Bahia, no qual a maioria dos profissionais não tinha hábito de praticar atividade física ${ }^{20}$. Fato preocupante, devido a importância da prática regular de atividades físicas para evitar doenças cardiovasculares, além de possibilitar a redução do estresse ${ }^{21}$. Um dos grandes fatores que levam a esse cenário é a insatisfação com a renda, fazendo com que esses indivíduos tenham mais de um vínculo laboral, não tendo tempo hábil para a mesma ${ }^{22}$.

Cada pessoa possui seus anseios e desejos a serem realizados em núcleo familiar, por isso, uma renda familiar que supra necessidades é sempre almejada. Todavia, na área da saúde, por vezes, um único emprego não é capaz de cumprir essa função, ocasionando então a dupla jornada de trabalho. Vemos isto no resultado deste estudo, pois $12,0 \%$ relataram ter mais de um vínculo empregatício, sendo que $11,0 \%$ o realizavam no período noturno. Com isto, tem-se o questionamento se esse trabalho excessivo faz com que essas pessoas deem menos atenção à sua própria saúde física e psicológica, resultando em uma má qualidade de vida $^{22}$. O trabalho noturno pode ainda acarretar em vários sintomas diferenciados da normalidade, pois há a mudança do padrão de 
sono, que pode desencadear aumento da irritabilidade e o baixo nível de alerta9 9

Além de sobrecarga laboral, observou-se a presença de doenças crônicas como a HAS, considerada um grave problema de saúde pública, considerando que a mesma ser responsável pelo desencadeamento de vários outros agravos de saúde, e está inteiramente relacionada a hábitos de vida, por isso a necessidade de uma alimentação adequada evitando o sódio, ingesta hídrica recomendada, e também a prática da atividade física. Em 2011 esta doença foi autorreferida por $22,7 \%$ de adultos brasileiros ${ }^{23}$. Em outro estudo realizado na Bahia com a população em geral, esta mesma patologia foi a mais citada ${ }^{24}$.

Quando questionados sobre o uso da medicação para a saúde mental, verificou-se que é uma prática aderida pela minoria em 31,5\%, percentual semelhante ao estudo realizado no estado de Alagoas, onde 37,4\% faziam uso de fármacos tranquilizantes e ansiolíticos ${ }^{25}$. Em um estudo realizado em Santa Catarina, demonstrou-se que a classe medicamentosa de saúde mental mais utilizada foi o antidepressivo em 47,1\%, assim como nesta pesquisa, entretanto, com uma taxa de $80,9 \%$ dos $\operatorname{casos}^{26}$.
Entre as medicações predominou a Fluoxetina com 26,9\%, corroborando o estudo realizado no meio Oeste de Santa Catarina, no qual este medicamento se encontrou em primeiro lugar de uso da população em geral, em 35,0\% dos casos. Ainda no mesmo estudo, a Sertralina apareceu em quarto lugar de uso com $21,7 \%$ dos casos, dado divergente do presente estudo, onde a mesma foi a segunda mais citada, com $25,3 \%{ }^{26}$

Apesar de o uso de medicação psicotrópica ter sido observado na minoria dos pesquisados, considera-se tal achado preocupante, pois esta minoria a utiliza mesmo conhecendo a característica de dependência da mesma, além dos efeitos esperados e adversos. Esses profissionais possuem fácil acesso a esses fármacos, devido ao seu meio laboral, o que pode levar ao uso mais frequente, e muitas vezes sem indicação médica para isto 9 .

$\mathrm{Na}$ atualidade é crescente o número de casos de depressão, por consequência, também o uso dos fármacos para controle dos sintomas da patologia. Sabe-se que existem várias terapias alternativas para controle desta doença, no entanto, a terapia farmacológica é a mais utilizada. O gênero que mais faz uso da medicação é o sexo feminino, o que pode estar 
relacionado ao fato de as mulheres procurarem mais os serviços de saúde para atendimento, em comparação com os homens. E com a grande maioria fazendo uso de medicação, temos a concepção de que muitos outros necessitam da mesma $^{27}$.

Ao avaliar a classe profissional que mais utilizava esse tipo de fármaco, houve predomínio de ACS e ACE com 47,6\%, divergente do estudo realizado na cidade de Montes Claros, MG, em que apenas 25,4\% faziam uso de medicação ${ }^{21}$. Um estudo realizado no estado de Minas Gerais retratou que os ACS pesquisados estavam esgotados emocionalmente, relacionando esse problema ao fato de os mesmos atuarem por um longo período de tempo nessa profissão, tendo como uma grande consequência, o uso de fármacos para controle de sinais e sintomas ${ }^{28}$.

Pereira retrata, ainda, em seu estudo que $29,1 \%$, da equipe de enfermagem fazia uso de medicação antidepressiva, ao passo que nesse estudo $12,6 \%$ dos técnicos de enfermagem faziam uso e $7,9 \%$ dos enfermeiros, valendo ressaltar que os mesmos têm o conhecimento dos efeitos das medicações. $O$ trabalho de enfermagem é complexo e exaustivo, com inúmeros agentes estressores que podem desencadear doenças psíquicas e interferir no estabelecimento de vínculos com o paciente, essencial ambos os envolvidos ${ }^{9}$.

A qualidade de vida é determinada pelos fatores predisponentes na vida individual da pessoa. Ela pode sofrer várias interferências como de patologias, de hábitos de vida e também de uso das medicações. Sabe-se que para uma boa qualidade de vida a satisfação do indivíduo tem de estar presente em todas as áreas da sua vida. O bem-estar no meio laboral, em sua saúde, vida física e psicológica, precisam estar presentes para um resultado satisfatório ${ }^{17}$.

A auto conceituação sobre qualidade de vida por vezes é limitada. No entanto, nesta pesquisa, vemos que a caracterização citada pelo público participante sobre a sua qualidade de vida assemelha-se às médias obtidas nos outros domínios. Isso se refere ao fato de as pessoas terem uma concepção adequada e fidedigna da sua vida e de como a mesma se encontra ${ }^{10}$.

Estudo realizado no Rio Grande do Sul os participantes relataram aspectos da qualidade de vida que os enquadraram no domínio psicológico com pontuação de 73,48 e o domínio físico com pontuação 80,17 (maior pontuação). $\mathrm{Na}$ avaliação do domínio meio ambiente, o mesmo teve uma média de 73,58 , e o social, $73,58^{6}$. Já estudo realizado em um município do 
interior de São Paulo, o qual contou com a participação de 98 profissionais que atuavam em ESF, demonstrou que a classificação dos mesmos para a sua autoavaliação de QV resultou em "boa", divergindo do resultado encontrado no presente estudo, onde a maioria dos profissionais classificou a sua QV como "regular". Nos demais domínios, ambos os estudos apresentaram como maior resposta, a classificação regular ${ }^{10}$.

A qualidade de vida está relacionada com o próprio meio laboral e a vida pessoal de cada indivíduo. Em virtude da carga horária diária, das cobranças na realização do serviço e obtenção de metas, faz com que o trabalho se torne exaustivo, e junta-se a isso o desânimo, o que leva a uma queda nos scores de qualidade de vida. Acrescente-se, também, que o cansaço adquirido no meio laboral persiste em sua vida privada, neste sentido, o profissional vai até sua casa, mas não tem o interesse da prática da atividade física, ou até mesmo de uma atividade de lazer 6 .

Trabalhar na área da saúde demanda uma vasta preparação psicológica dos profissionais, pois os mesmos estão constantemente expostos a situações problemáticas dos pacientes, portanto, necessitando de conhecimento e discernimento para saber lidar com a problematização do cliente, buscar soluções e formas de auxílio para o mesmo, o que demanda do profissional tempo, para obter a esperada resolutividade ${ }^{10}$.

Nas médias obtidas no presente estudo, a mais baixa foi a de saúde física, e isso está relacionado ao fato de a maioria dos participantes ser mulher, cuja característica de vida é de dupla jornada de trabalho, pois mulheres, além de profissionais são donas de casa, mães e esposas. Com isso, elas sentem-se mais cansadas, e, consequentemente sua área física é afetada ${ }^{29}$.

Ao avaliar a faceta meio ambiente temos uma média regular, isto se assemelha a outros estudos, demonstrando que as atividades de lazer, segurança e saúde não estão de acordo com o esperado ${ }^{17}$. O mesmo está relacionado ainda ao acesso a novas informações e novas qualificações, no entanto, com a média obtida, percebe-se que o mesmo também é falho ${ }^{6}$.

As relações socias, com a maior média, está relacionado ao apoio social, às amizades, à convivência com as pessoas/colegas no dia a dia. E relaciona ainda, a atividade sexual, a qual é uma necessidade fisiológica, descrita por Wanda Aguiar Horta ${ }^{29}$. 
A avaliação da qualidade de vida é algo muito subjetivo, considerando que cada indivíduo possui uma percepção diferenciada. Com isso, a avalição da mesma possui limitações, no entanto, nada que impeça o resultado final. A aplicação do questionário ocorreu no horário de trabalho dos profissionais, com isso, há a possibilidade de alguns não estarem completamente concentrados em seu preenchimento.

\section{Conclusão}

Conclui-se com esta pesquisa, que os profissionais da Atenção Primária em Saúde se encontravam com uma má QV, e esse conceito não modifica sua autoavaliação. Junto a isto, percebe-se um número significativo desta população com doenças crônicas associadas em sua vida diária. Diante de tal panorama, julga-se necessária a elaboração de políticas públicas voltadas para esses profissionais, objetivando uma melhora deste parâmetro de QV.

Visualiza-se também com este estudo, um grande número de profissionais de saúde que fazem uso de medicação psicotrópica, principalmente antidepressivos, associado ao sedentarismo e pouca atividade de lazer. Tem-se a necessidade da aplicação de outras terapias alternativas, combinada com psicoterapia, rodas de conversa, essenciais para a melhora destes resultados.

\section{Referências}

1. World Health Organization (WHO) (Homepage na internet). WHOQOL: Medindo a Qualidade de Vida (Acesso em 04 out 2019). Disponível em: https://www.who.int/.

2. Silva RF, Silva SF, Barbosa TC, Quaresma FRP, Maciel ES. Nível de Percepção de Estresse e Qualidade de Vida Entre os Técnicos de Enfermagem das Unidades de Pronto Atendimento de Palmas - TO. R bras ci Saúde. 2018;22(3):261-266.

DOI: 10.4034/RBCS.2018.22.03.10.

3. Neves DR, Nascimento RP, Felix Jr MS, Silva FA, Andrade ROB. Sentido e significado do trabalho: uma análise dos artigos publicados em periódicos associados à Scientific Periodicals Electronic Library. Caderno ABAPE.BR. 2018;16(2):318-330. DOI: 10.1590/1679395159388 .

4. Anais da VIII Jornada de Políticas Públicas;22-25 ago 2017; São Luís (MA): UFMA; 2017.

5. Dias EG, Santos AR, Souza ELS, Araújo MML, Alves JCS, Mishima SM. Qualidade de vida no trabalho dos profissionais da saúde de uma Unidade Básica de Saúde. Revista Cubana de Enfermería. 2016;32(4):126-137.

6. Ferigollo JP, Fedosse E, Santos-Filha VAV. Qualidade de vida de profissionais da Saúde Pública. Cad. Ter. Ocup. 2016;24(3):497-507. DOI: 10.4322/0104-4931.ctoAO0722.

7. Andrade KO, Andrade PO, Leite LF. Qualidade de Vida dos trabalhadores da área da Saúde: Revisão de Literatura. Revista Científica do ITPAC. 2015;8(1):1-4.

8. Roque H, Veloso A, Silva I, Costa P. Estresse ocupacional e satisfação dos usuários com os cuidados de saúde primários em Portugal. 
Ciência \& Saúde Coletiva. 2015;20(10):30873097. DOI: $10.1590 / 1413$

812320152010.00832015 .

9. Pereira IF, Faria LC, Vianna RSM, Corrêa PDS, Freitas DA, Soares WD. Depressão e uso de medicamentos em profissionais de enfermagem. Arq. Ciênc. Saúde. 2017;24(1):7074. DOI: 10.17696/2318-3691.24.1.2017.544.

10. Gomes MFP, Mendes ES, Fracolli LA. Qualidade de Vida dos Profissionais que trabalham na Estratégia Saúde da Família. Rev. Aten. Saúde. 2016;14(48):27-33. DOI: 10.13037/rbcs.vol14n49.3695.

11. Fleck MPA, Louzada S, Xavier $M$, Chachamovich E, Vieira G, Santos L, et al. Aplicação da Versão em Português do Instrumento Abreviado de Avaliação da Qualidade de Vida "WHOQOL-bref". Rev Saúde Pública. 2000;34(2):178-183. DOI: 10.1590/S0034-89102000000200012.

12. Carlotto MS. Transtornos Mentais Comuns em trabalhadores de Unidades Básicas de Saúde: Prevalência e fatores associados. PsicolArgum. 2016;34(85):133-146. DOI:

10.7213/psicol.argum.34.085.AO04.

13. Moreira IJB, Horta JA, Duro LN, Borges DT, Cristofari AB, Chaves $J$ et al. Perfil sociodemográfico, ocupacional e avaliação das condições de saúde mental dos trabalhadores da Estratégia Saúde da Família em um município do Rio Grande do Sul, RS. Rev Bras Med Fam Comunidade. 2016;11(38):1-12. DOI: $10.5712 /$ rbmfc11(38)967.

14. Leonelli LB, Andreoni S, Martins P, Kozasa EH, Salvo VL, Sopezki D et al. Estresse percebido em profissionais da Estratégia Saúde da Família. Rev Bras Epidemiol. 2017;20(2):286-298. DOI: 10.1590/19805497201700020009 .

15. Brasil. Constituição (1998). Emenda constitucional n. ${ }^{\circ} 51$, de 14 de fevereiro de 2006. Dá nova redação ao art. 198 da Constituição Federal, alternando e inserindo parágrafos. fev 2016.

16. Nunes EFPA, Santini SML, Carvalho BG, Cordoni-Junior L. Força de trabalho em saúde na Atenção Básica em Municípios de Pequeno
Porte do Paraná. Saúde Debate. 2015;39(104):29-41. DOI: 10.1590/0103110420151040174 .

17. Freire MN, Costa ER, Alves EB, Santos CMF, Santos CO. Qualidade de Vida dos profissionais de Enfermagem no ambiente laboral hospitalar. Rev enferm UFPE on line. 2016;10(6):4286-4294. DOI: 10.17267/23173378rec.v5i1.871.

18. Instituto Brasileiro de Geografia e Estatística [homepage na internet]. Características gerais da população, religião e pessoas com deficiência. (Acesso em 04 out 2019). Disponível em: http:www.ibge.gov.br.

19. Guimarães MSA, Sousa MF, Mucari TB. Perfil sociodemográfico dos agentes comunitários de Saúde da Estratégia Saúde da Família no município de Palmas-TO. Revista Desafios. 2017;04(03):60-72. DOI: 10.20873/uft.2359-3652.2017v4n3p60.

20. Carvalho DB, Araújo TM, Bernardes KO. Transtornos mentais comuns em trabalhadores da Atenção Básica à Saúde. Rev Bras Saude Ocup. 2016;41(17):1-13. DOI: 10.1590/23176369000115915.

21. Santos TI, Guerra PH, Andrade DR, Florindo AA. Práticas pessoais e profissionais de promoção da atividade física em agentes comunitários de saúde. Rev Bras Ativ Fís Saúde. 2015;20(2):165-173.

DOI: 10.12820/rbafs.v.20n2p165.

22. Albuquerque GA, Nunes JFC, Belém JM, Leite MF, Quirino GS. Dupla jornada de trabalho: implicações na Saúde da Enfermeira. Rev enferm UFPE on line. 2016;10(9):34013410. DOI: 10.5205/reuol.9571-83638-1SM1009201628.

23. Bloch KV, Klein CH, Szklo M, Kuschnir MCC, Abreu GA, Barufaldi LA et al. ERICA: prevalências de hipertensão arterial e obesidade em adolescentes brasileiros. Rev de Saúde Pública. 2016;50(1):1-13. DOI:10.1590/S01518-8787.2016050006685.

24. Almeida-Brasil CC, Silveira MR, Silva KR, Lima MG, Faria CDCM, Cardoso CL et al. Qualidade de vida e características associadas: aplicação do WHOQOL-BREF no contexto da 
Atenção Primária à Saúde. Ciência \& Saúde Coletiva. 2017;22(5):1705-1716. DOI: 10.1590/1413-81232017225.20362015.

25. Maciel MPGS, Santana FL, Martins CMA, Costa WT, Fernandes LS, Lima JS. Uso de medicamentos psicoativos entre profissionais de saúde. Rev enferm UFPE on line. 2017;11(7):2881-2887.

10.5205/reuol.11007-98133-3-

SM.1107sup201709.

26. Braga DC, Bortolini SM, Pereira TG, Hildebrando RB, Conte TA. Uso de psicotrópicos em um município do meio oeste de Santa Catarina. J Health Sci Inst. 2016;34(2):108-113.

27. Soares SB, Muniz SDB, Albuquerque FGF, Malaquias IS, Leite FC. Avaliação de uso de antidepressivos em uma farmácia privada na cidade de Cajazeiras-PB. Journal of Biology \& Pharmacy and Agricultural Management. 2019;15(3):282-294.

28. Silva MA, Lampert SS, Bandeira DR, Bosa CA. Saúde emocional de Agentes Comunitários: burnout, estresse, bem-estar e qualidade de vida. Revista da SPAGESP. 2017;18(1):20-33.

29. Mujchin IG. Quality of Life of the Health Care Workers in the Pre-Retirement Period from the Private Sector of the Primary Health Care from the Skopje Region. OA Maced J Med Sci. 2015;3(3):514-520.

DOI:
Agradecimentos -agradecimentos a colaboradores entre outros poderão ser citados nesta seção, antes das referências.

Financiamento - citar as fontes de recursos de financiamento em ordem alfabética

Anexos - espaço para documentos importantes para o estudo, ex. instrumentos de coleta, tabelas de dados, análises estatísticas complementares, etc

Os Anexos podem ser enviados como documentos suplementares durante o processo de submissão na página da revista AES

Reservado aos Editores

Data de submissão: 23/09/2020

Data de aprovação:08/12/2020

10.3889/oamjms.2015.097. 